\title{
History of cardiovascular events and cardiovascular risk factors among patients initiating strontium ranelate for treatment of osteoporosis
}

This article was published in the following Dove Press journal:

International Journal of Women's Health

17 November 2015

Number of times this article has been viewed

\author{
Jingbo $\mathrm{Yu}^{\prime}$ \\ Jackson Tang ${ }^{2}$ \\ Zhiyi Li ${ }^{2}$ \\ Shiva Sajjan' \\ Christopher O'Regan ${ }^{3}$ \\ Ankita Modi' \\ Vasilisa Sazonov' \\ 'Center for Observational and \\ Real-World Evidence (CORE), \\ Merck \& Co., Inc., Kenilworth, NJ, \\ USA; ${ }^{2}$ Asclepius Analytics Ltd., \\ Wanchai, Hong Kong; ${ }^{3}$ Merck Sharp \\ \& Dohme Corp., Hoddeson, UK
}

Correspondence: Ankita Modi Center for Observational and RealWorld Evidence (CORE), Merck \& Co., Inc., Supporting Bone Products, Mailstop CRB-205, Kenilworth, NJ, USA

Tel +I 9082364563

Email ankita.modi@merck.com
Purpose: To estimate the proportion of osteoporosis patients in whom initiating strontium ranelate treatment, under new EMA guidelines, should be contraindicated because of a history of cardiovascular events or risk for cardiovascular events.

Materials and methods: This was a retrospective analysis of medical and pharmacy claims using the Clinical Practice Research Datalink database. Patients were included if they had $\geq 1$ prescription of strontium from September 1, 2008 to August 31, 2013, were aged $\geq 50$ as of the index date (the date of the first ever strontium ranelate prescription), and had $\geq 1$ year of medical records pre-index. Cardiovascular events occurring any time pre-index were identified, which included ischemic heart disease, cerebrovascular disease, uncontrolled hypertension, and peripheral arterial disease. Cardiovascular risk factors assessed included 1) diabetes or hypertension any time pre-index; 2) hyperlipidemia in the 12 months pre-index; or 3) obesity in the 12 months pre-index.

Results: A total of 7,474 patients were included: $90.4 \%$ were women, with an average age of 76.5 years, and $84.5 \%$ used osteoporosis therapy, either bisphosphonates or non-bisphosphonates, prior to strontium initiation. A total of $23.6 \%$ of patients experienced $\geq 1$ cardiovascular event prior to strontium initiation; the rate was lower among female patients than in male patients $(22.4 \%$ vs $35.3 \%, P<0.01)$. A total of $45.9 \%$ had risk factors for cardiovascular events (without cardiovascular event history).

Conclusion: More than one-fifth of osteoporosis patients in the UK who used strontium had a cardiovascular event history, and one-half had cardiovascular risk factors prior to strontium initiation.

Keywords: osteoporosis, cardiovascular diseases, risk of cardiovascular event, drug therapy, drug safety

\section{Introduction}

Strontium ranelate is a relatively new drug used for the treatment of osteoporosis that both stimulates bone formation and inhibits bone resorption. ${ }^{1,2}$ Randomized, placebocontrolled clinical trials have demonstrated the efficacy of strontium ranelate in reducing the risk of osteoporotic vertebral and nonvertebral fractures. ${ }^{1-3}$ Strontium ranelate was first approved for use in the European Union in 2004. ${ }^{4}$ Guidelines from the UK's National Institute for Health and Clinical Excellence describe strontium ranelate as an alternative treatment option for the prevention of osteoporotic fragility fractures in postmenopausal women who are unable to comply with the special instructions for the administration of bisphosphonates, namely alendronate and either risedronate or etidronate. ${ }^{5}$

The safety profile of strontium ranelate was similar to that of placebo in individual clinical trials, but in recent pooled analyses conducted by the European Medicines 
Agency (EMA), increased incidences of venous thromboembolism and myocardial infarction (MI) have been observed..$^{3,4}$ Although the use of strontium was shown to have no effect on the incidence of cardiac events in observational studies, ${ }^{6,7}$ in 2014, the EMA, based on the pooled clinical trial data, restricted the use of strontium to patients who cannot be treated with other medicines approved for osteoporosis. The EMA also recommended the regular evaluation of cardiovascular conditions in patients using the medication and advised that patients with a history of cardiovascular disease (CVD) (ie, ischemic heart disease [IHD], cerebrovascular disease, uncontrolled hypertension, or peripheral artery disease $[\mathrm{PAD}])$ not use strontium. ${ }^{8-10}$

In light of these recent changes, it is important to know the percentage of osteoporosis patients to whom the EMA recommendations for strontium apply. The only such information available is from a retrospective cohort study in Denmark, conducted during 2005-2007, which assessed the presence of CVD and cardiovascular risk factors in patients receiving strontium ranelate. ${ }^{11}$ The objective of the current analysis was to estimate the proportion of osteoporosis patients with a history of cardiovascular events or cardiovascular risk factors who were prescribed strontium ranelate in the UK during 2008-2013. A secondary objective was to quantify the proportion of patients who had used two different bisphosphonate medications (ie, alendronate and either risedronate or etidronate) prior to starting strontium ranelate, as described in the NICE guidelines.

\section{Materials and methods Study design}

This was a retrospective analysis of medical and pharmacy claims using data derived from the UK Clinical Practice Research Datalink (CPRD) database for years 2000-2013. Subjects were included if they newly initiated treatment with strontium ranelate between September 1, 2008 and August 31, 2013 (defined as the index period). This index period was chosen to provide an overview of the cardiovascular events and risk factors in patients initiating strontium ranelate in the most recent 5 years at the time of the analysis. The index date was defined as the date of the first prescription for strontium ranelate during the index period. Cardiovascular events and risk factors occurring any time before the index date were identified in the patients through the database.

\section{Study sample}

Study subjects were men and women at least 50 years of age as of the index date, who had received at least one prescription for strontium ranelate during the index period, and who had data for at least 1 year prior to the index date. Patients with an index date $<12$ months after the up-tostandard date (ie, the date on which the participating practice met the CPRD standards), or $>1$ osteoporosis therapy on the index date, or a history of malignant neoplasm (except nonmelanoma skin cancer) or metabolic bone disease (including rickets or osteomalacia, hyperparathyroidism, or Paget's disease of bone) any time before or on the index date were excluded from the analysis. These criteria ensure a 12-month period in which to evaluate CVD history, receipt of strontium as monotherapy, and receipt of strontium for osteoporosis (as opposed to another bone disease), respectively. The patient data were de-identified, so informed consent was not required. The study protocol was approved by the Independent Scientific Advisory Committee in CPRD in December 2013.

\section{Study variables}

Cardiovascular events were defined as diagnoses of IHD, cerebrovascular disease, PAD, or occurrences of uncontrolled hypertension at any time prior to the index date. IHD included MI, angina (angina pectoris), and chronic IHD. Cerebrovascular diseases included stroke (ischemic stroke, cerebral thrombosis, or cerebral hemorrhage), cerebral embolism, transient ischemic attack, cerebral atherosclerosis, cerebral ischemia, and cerebral infarction. Uncontrolled hypertension was defined as $>140 / 90 \mathrm{mmHg}$ and was determined from the mean value of the two most recent blood pressure measurements prior to the index date.

Cardiovascular risk factors were defined as follows: 1) diagnoses or treatment of hypertension or type 2 diabetes at any time prior to the index date; 2) a diagnosis of hyperlipidemia or treatment with statins in the year prior to the index date; or 3) a diagnosis of obesity or a body mass index (BMI) indicating obesity in the year prior to the index date. Obesity was defined as a BMI of $\geq 30 \mathrm{~kg} / \mathrm{m}^{2}$ and was determined from the most recent measurement before the index date.

The prescription of strontium ranelate was identified using multiplex codes. Cardiovascular events, risk factors, and other morbid conditions, such as osteoporosis and fracture, were identified using Read codes in the CPRD database.

\section{Statistical analysis}

The demographic and clinical characteristics of the study population were analyzed descriptively. Continuous 
variables were described by their means and standard deviations and categorical variables by their frequency (number and percentage). Two subgroups were defined and analyzed similarly: patients with a history of a cardiovascular event and patients with cardiovascular risk factors but no cardiovascular event in their history. Patients with a confirmed diagnosis of osteoporosis prior to the index date were assessed by a sensitivity analysis to ensure that the presence or absence of the diagnosis code did not affect the overall study results.

The frequencies of cardiovascular events and cardiovascular risk factors were tabulated for the combined study population and for men and women separately. The same results were reported for patients with a prior osteoporosis diagnosis, by a sensitivity analysis. Differences in cardiovascular events and risk factors between men and women were assessed for statistical significance using the $\chi^{2}$ test, and continuous variables such as age were assessed for statistical significance using Student's $t$-tests. A $P$-value $<0.05$ was considered statistically significant.

\section{Results}

\section{Characteristics of the study population}

A total of 7,474 subjects met the inclusion criteria (Figure 1). The mean age was 76.5 years (76.5 for women and 75.9 for men), $90.4 \%$ were women, $63.5 \%$ had a prior diagnosis of osteoporosis, and $44.3 \%$ had a history of osteoporosis-related fracture (Table 1). A majority of patients (72.4\%) had a history of bisphosphonate use, and $27.2 \%$ had used two or more different bisphosphonates before initiating strontium ranelate (Table 1).

Sensitivity analyses showed that the subgroup of patients with a diagnosis of osteoporosis $(\mathrm{N}=4,749)$ was numerically similar to the total study population in mean age ( 75.0 years), percentage of women (93.4\%), and history of osteoporosisrelated fracture $(42.6 \%)$.

\section{History of cardiovascular events}

Cardiovascular events had previously occurred in 1,767 of the 7,474 subjects who initiated strontium ranelate $(23.6 \%$; Table 2). Among these subjects, a diagnosis of IHD was the most common type of event (13.1\%), followed by diagnoses of cerebrovascular disease $(8.3 \%)$ and uncontrolled hypertension $(3.3 \%)$. For all types of cardiovascular events except uncontrolled hypertension, men were significantly more frequently diagnosed than women, and $35.3 \%$ of all men versus $22.4 \%$ of all women had a history of any cardiovascular event $(P<0.01)$.
10,272

Patients receiving a prescription of strontium ranelate during the study period

(September 1, 2008-August 31, 2013)

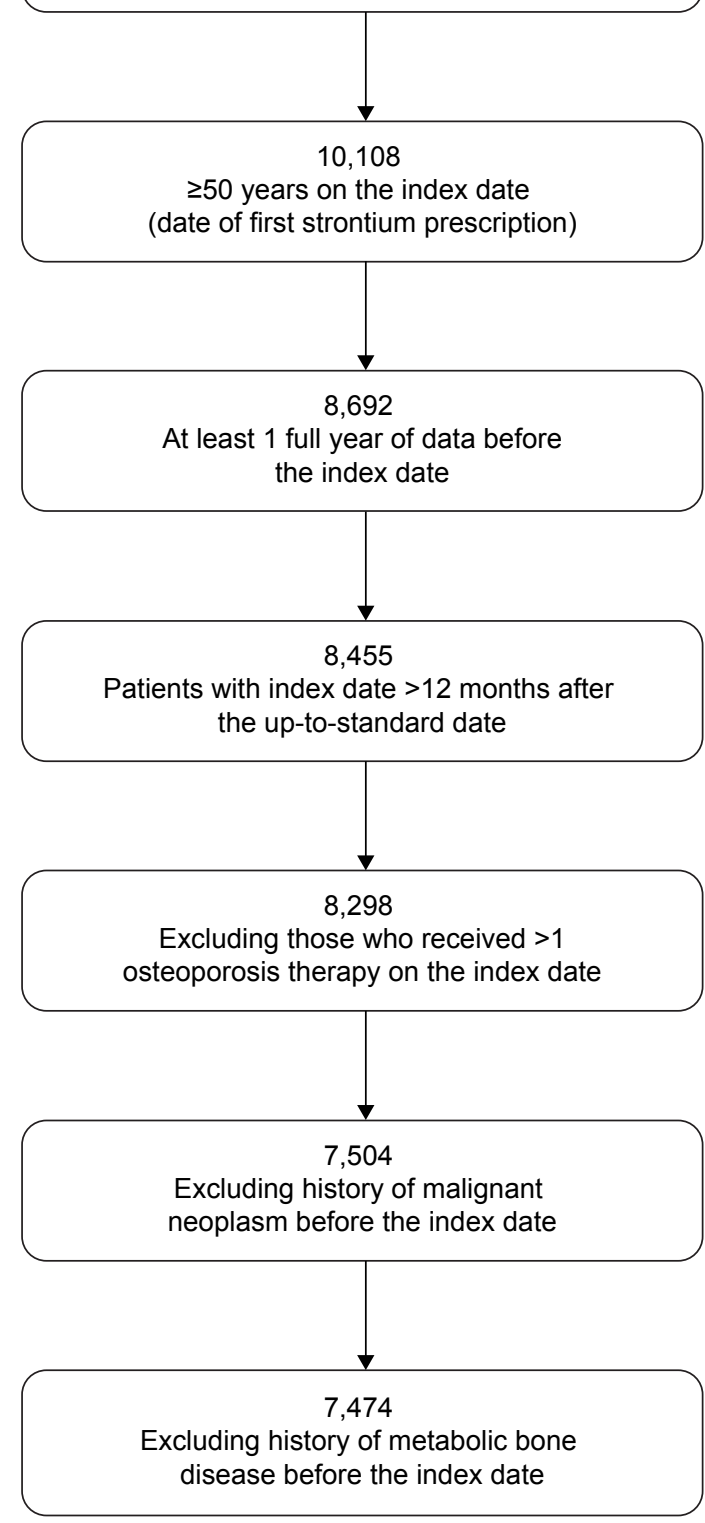

Figure I Patient selection.

In sensitivity analyses of the patients with an osteoporosis diagnosis, the frequency of cardiovascular events (20.8\%) and their categorical distribution (IHD, 11.8\%; cerebrovascular diseases, 7.0\%; uncontrolled hypertension, $3.1 \%$; PAD, $1.8 \%$ ) were numerically similar to the total study population.

\section{History of cardiovascular risk factors}

A total of 3,431 patients initiating strontium ranelate $(45.9 \%)$ had one or more cardiovascular risk factors without having had a cardiovascular event (Table 3 ). In this subgroup, the 
Table I Patient characteristics ${ }^{\mathrm{a}}$

\begin{tabular}{|c|c|}
\hline Characteristic & Total $(\mathrm{N}=\mathbf{7 , 4 7 4})$ \\
\hline Age, years (mean, SD) & $76.5(10.3)$ \\
\hline \multicolumn{2}{|l|}{ Age, years } \\
\hline $50-59$ & $491(6.6)$ \\
\hline $60-69$ & $\mathrm{I}, 485(19.9)$ \\
\hline $70-79$ & $2,25 I(30.1)$ \\
\hline$\geq 80$ & $3,247(43.4)$ \\
\hline \multicolumn{2}{|l|}{ Sex } \\
\hline Male & $714(9.6)$ \\
\hline Female & $6,760(90.4)$ \\
\hline \multicolumn{2}{|l|}{ Index year } \\
\hline 2008 & $\mathrm{I}, 777(23.8)$ \\
\hline 2009 & $\mathrm{I}, 465(19.6)$ \\
\hline 2010 & $\mathrm{I}, 273(17.0)$ \\
\hline 2011 & $1,245(16.7)$ \\
\hline 2012 & $\mathrm{I}, 124(15.0)$ \\
\hline 2013 & $590(7.9)$ \\
\hline \multicolumn{2}{|l|}{ Comorbidities $^{\mathrm{b}}$} \\
\hline Hyperparathyroidism & $12(0.2)$ \\
\hline Hypothyroidism & $68(0.9)$ \\
\hline Hyperthyroidism & $16(0.2)$ \\
\hline Chronic inflammatory bowel disease & $2(0.0)$ \\
\hline Celiac disease & $8(0.1)$ \\
\hline Vitamin D deficiency & $30(0.4)$ \\
\hline Depression/anxiety & $132(1.8)$ \\
\hline Alzheimer's disease & $35(0.5)$ \\
\hline Parkinson's disease & $28(0.4)$ \\
\hline \multicolumn{2}{|l|}{ History of OP-related events ${ }^{c}$} \\
\hline Diagnosis of OP & $4,749(63.5)$ \\
\hline \multicolumn{2}{|l|}{ OP medication use } \\
\hline Bisphosphonate use & $5,414(72.4)$ \\
\hline$\geq 2$ different bisphosphonates & $2,030(27.2)$ \\
\hline Non-bisphosphonate use & $3,195(42.7)$ \\
\hline None & $\mathrm{I}, 155(15.5)$ \\
\hline OP-related fracture, past 12 months & I,324 (I7.7) \\
\hline Hip & $719(9.6)$ \\
\hline Vertebral & $133(1.8)$ \\
\hline Nonhip, nonvertebral & $548(7.3)$ \\
\hline
\end{tabular}

Notes: ${ }^{a}$ Values are presented as $\mathrm{N}$ (\%) unless otherwise indicated; bbased on diagnostic codes occurring within the past 12 months; 'categories of osteoporosis medication use and osteoporosis-related fractures were not mutually exclusive.

Abbreviations: OP, osteoporosis; SD, standard deviation. proportion of men and women were statistically similar (43.3\% and $46.2 \%$, respectively; $P=0.14)$. The most common risk factor was hypertension (38.5\%), followed by hyperlipidemia (18.6\%), obesity ( $8.3 \%)$, and type 2 diabetes (4.7\%; Table 3). The proportion of men and women with hypertension and diabetes were numerically similar $(P=0.80$ and 0.23 , respectively), but hyperlipidemia and obesity were more common in women (hyperlipidemia: $18.9 \%$ of women versus $15.8 \%$ of men; obesity: $8.6 \%$ of women versus $5.6 \%$ of men; $P=0.04$ and $<0.01$, respectively; Table 3).

Similar to the results obtained earlier, sensitivity analyses of patients with an osteoporosis diagnosis showed that the frequency of cardiovascular risk factors (45.3\%) and their categorical distribution (hypertension, 37.3\%; hyperlipidemia, $19.2 \%$; obesity, $7.4 \%$; type 2 diabetes, $3.6 \%$ ) were numerically similar to the total study population.

\section{Discussion}

This study showed that $24 \%$ of patients initiating strontium ranelate for the treatment of osteoporosis during 2008-2013 had a history of cardiovascular events and an additional $45 \%$ had one or more cardiovascular risk factors. To our knowledge, only one study has previously assessed the presence of CVD and cardiovascular risk factors in patients receiving strontium ranelate. ${ }^{11}$ Abrahamsen et al reported the prevalence of cerebrovascular disease (11.3\%), peripheral vascular disease (6.2\%), and MI (6.8\%) among users of strontium ranelate in Denmark (mean age 74.0 years, $11.8 \%$ male). ${ }^{11}$ The combined prevalence of MI, peripheral vascular disease, and cerebrovascular disease in the study by Abrahamsen et al (19.2\% in females and $29.5 \%$ in males) is similar to the combined prevalence of MI, PAD, and cerebrovascular disease observed in our study population (14.2\% and 27.4\%, respectively; Table 2). Prescriptions for antihypertensive medications, statins, and diabetes

Table 2 History of cardiovascular events ${ }^{\mathrm{a}}$

\begin{tabular}{|c|c|c|c|c|}
\hline Cardiovascular event & Total $(\mathbf{N}=7,474)$ & $\operatorname{Men}(N=7 \mid 4)$ & Women $(\mathrm{N}=6,760)$ & $P$-value ${ }^{b}$ \\
\hline Any event & I,767 (23.6) & $252(35.3)$ & I,5I5 (22.4) & $<0.01 *$ \\
\hline Ischemic heart disease & $977(13.1)$ & $147(20.6)$ & $830(12.3)$ & $<0.0 I^{*}$ \\
\hline Angina & $792(10.6)$ & $118(16.5)$ & $674(10.0)$ & $<0.01 *$ \\
\hline Myocardial infarction & $380(5.1)$ & $76(10.6)$ & $304(4.5)$ & $<0.01 *$ \\
\hline Cerebrovascular diseases & $623(8.3)$ & $88(12.3)$ & $535(7.9)$ & $<0.01 *$ \\
\hline Stroke & $595(8.0)$ & $82(11.5)$ & $513(7.6)$ & $<0.01 *$ \\
\hline Cerebral infarction & $50(0.7)$ & $10(1.4)$ & $40(0.6)$ & $0.01 *$ \\
\hline Uncontrolled hypertension & $244(3.3)$ & $27(3.8)$ & $217(3.2)$ & 0.41 \\
\hline Peripheral artery disease & $157(2.1)$ & $32(4.5)$ & $125(1.8)$ & $<0.0 I^{*}$ \\
\hline
\end{tabular}

Notes: Event categories are nonmutually exclusive. ${ }^{2}$ Values are presented as $\mathrm{N}$ (\%); ${ }^{b}$-values are for the comparison between men and women; *indicates statistical significance. 
Table 3 Presence of cardiovascular risk factors ${ }^{\mathrm{a}}$

\begin{tabular}{|c|c|c|c|c|}
\hline Risk factor & Total $(\mathbf{N}=\mathbf{7 , 4 7 4})$ & $\operatorname{Men}(N=7 \mid 4)$ & Women $(\mathrm{N}=6,760)$ & $P$-value ${ }^{b}$ \\
\hline Any risk factor & 3,43 I (45.9) & $309(43.3)$ & $3,122(46.2)$ & 0.14 \\
\hline Hypertension & $2,880(38.5)$ & $272(38 . I)$ & $2,608(38.6)$ & 0.80 \\
\hline Hyperlipidemia & I,392 (18.6) & $113(15.8)$ & $1,279(18.9)$ & $0.04^{*}$ \\
\hline Obesity & $624(8.3)$ & $40(5.6)$ & $584(8.6)$ & $<0.0 I^{*}$ \\
\hline Type 2 diabetes & $35 \mid(4.7)$ & $40(5.6)$ & $3 I \mid(4.6)$ & 0.23 \\
\hline
\end{tabular}

Notes: Cardiovascular risk was tabulated only for patients without a history of cardiovascular events. Risk categories are nonmutually exclusive. ${ }^{a} \vee a l u e s$ are presented as $\mathrm{N}$ (\%); ${ }^{\mathrm{P}} \mathrm{P}$-values are for the comparison between men and women, *indicates statistical significance.

medications were received by $52.3 \%, 15.7 \%$, and $4.2 \%$ of the Danish study population in the year before initiating strontium ranelate. ${ }^{11}$ By comparison, $38.5 \%$ of our study population had hypertension, $18 \%$ had hyperlipidemia, and $4.7 \%$ had diabetes.

Only one observational study conducted in the UK had assessed cardiovascular outcomes in patients taking strontium ranelate. Cooper et al analyzed MI, hospitalization with MI, and cardiovascular death in 112,445 women with postmenopausal osteoporosis and found that the use of strontium was not associated with these outcomes. ${ }^{6}$ In their study population, $6.9 \%$ of the female recipients having strontium ranelate had previously had an acute ischemic cardiac event (MI, acute coronary syndrome, or unstable angina).

The baseline prevalence of cardiovascular events in the study by Cooper et al was quite low (6.9\%) compared to the prevalence in this study $(23.6 \%)$. This may be likely due to the differences in study design, primarily the uneven balance between the sexes in the study populations and the types of cardiovascular events included in the analysis, and our results must be interpreted in light of these factors. In the current study, $9.6 \%$ of the study population was male, and a history of cardiovascular events was more frequently observed in men than in women. Thus, the inclusion of men may have increased the overall prevalence of cardiovascular events. In addition, we included a broad array of cardiovascular events, both cardiac and cerebrovascular, in the analyses. Since the individual categories of cardiovascular events and risk factors were not mutually exclusive, the frequencies at which they occurred cannot simply be added to arrive at a total prevalence. However, the definitions of "any" cardiovascular event and "any" cardiovascular risk factors were designed to take this into account, and thus the overall prevalence estimates are an accurate representation of the prevalence of cardiovascular events and risk factors within the study population.

The primary caveat of the study design is the retrospective nature of the cardiovascular risk factor data. Some of the risk factors, eg, hypertension and hyperlipidemia, may have been controlled by the time strontium was initiated, but the analysis did not identify such patients. This may have led to the overestimation of the number of patients in whom the use of strontium would be contraindicated. Another limitation is that an osteoporosis diagnosis was not an inclusion criterion, and this weakens the applicability of our findings to patients with a confirmed diagnosis of osteoporosis. However, in our analyses of the subgroup of patients with an osteoporosis diagnosis, no substantial differences from the overall study population were observed. Similarly, we did not assess cardiovascular events and risk factors in relation to the presence of an osteoporotic fracture, so the results may not be directly applicable to patients with a fracture. Other minor limitations are those common to all administrative database analyses and include potential coding errors and incomplete data on cardiovascular risk factors, which may have affected patient classification.

Approximately, one-quarter of patients $(27.2 \%)$ in this study had already been treated with two or more different bisphosphonates before initiating strontium ranelate. This proportion was similar in patients with a history of cardiovascular events $(23.7 \%)$ and with a history of cardiovascular risk factors (26.3\%; data not shown). Since the EMA now recommends that patients with a history of cardiovascular events should not use strontium, alternative therapies will be needed to prevent osteoporotic fractures in this population.

\section{Conclusion}

In conclusion, according to the most recent recommendations of the EMA, approximately one-fourth of strontium ranelate users in the UK should not use this treatment due to a history of cardiovascular events, and almost half of these patients have at least one cardiovascular risk factor that should be monitored regularly by their physician.

\section{Acknowledgments}

The authors thank Anna Kaufman, MPH, and Melissa Stauffer, $\mathrm{PhD}$, in collaboration with SCRIBCO, for medical writing assistance. This study was funded by Merck \& Co., Inc. 


\section{Author contributions}

AM, COR, JY, SS, and VS conceived and designed the study. JT and ZL managed and analyzed the data. AM, JT, JY, SS, VS, and ZL interpreted the data. All authors contributed to drafting the article or revising it critically for important intellectual content. All authors approved the final version of this manuscript and agree to be accountable for all aspects of the work.

\section{Disclosure}

AM, SS, and VS are employees of Merck \& Co., Inc. JY was an employee of Merck \& Co., Inc. at the time of the study. COR is an employee of Merck Sharp \& Dohme Corp., a subsidiary of Merck \& Co., Inc., Kenilworth, NJ, USA. JT and ZL received financial remuneration from Merck \& Co., Inc. for conducting the study. The authors report no other conflicts of interest in this work.

\section{References}

1. Cesareo R, Napolitano C, Iozzino M. Strontium ranelate in postmenopausal osteoporosis treatment: a critical appraisal. Int $J$ Womens Health. 2010;2:1-6.

2. Cianferotti L, D'Asta F, Brandi ML. A review on strontium ranelate long-term antifracture efficacy in the treatment of postmenopausal osteoporosis. Ther Adv Musculoskelet Dis. 2013;5(3):127-139.

3. Stevenson M, Davis S, Lloyd-Jones M, Beverley C. The clinical effectiveness and cost-effectiveness of strontium ranelate for the prevention of osteoporotic fragility fractures in postmenopausal women. Health Technol Assess. 2007;11(4):1-134.
4. European Medicines Agency. PSUR assessment report: Strontium ranelate; 2013. Available from: http://www.ema.europa.eu/docs/ en_GB/document_library/EPAR_-_Assessment_Report_-_Variation/ human/000560/WC500147168.pdf. Accessed March 31, 2014.

5. National Institute for Health and Clinical Excellence. Alendronate, Etidronate, Risedronate, Raloxifene, Strontium Ranelate and Teriparatide for the Secondary Prevention of Osteoporotic Fragility Fractures in Postmenopausal Women (amended). London: National Health Service; 2008.

6. Cooper C, Fox KM, Borer JS. Ischaemic cardiac events and use of strontium ranelate in postmenopausal osteoporosis: a nested casecontrol study in the CPRD. Osteoporos Int. 2014;25(2):737-745.

7. Svanstrom H, Pasternak B, Hviid A. Use of strontium ranelate and risk of acute coronary syndrome: cohort study. Ann Rheum Dis. 2014; 73(6):1037-1043.

8. European Medicines Agency. Recommendation to Restrict the Use of Protelos/Osseor (strontium ranelate); 2013. Available from: http://www.ema.europa.eu/docs/en_GB/document_library/Press_ release/2013/04/WC500142507.pdf. Accessed April 11, 2014.

9. Donneau AF, Reginster JY. Cardiovascular safety of strontium ranelate: real-life assessment in clinical practice. Osteoporos Int. 2014;25(2):397-398.

10. European Medicines Agency. European Medicines Agency Recommends that Protelos/Osseor Remain Available but with Further Restrictions; 2014. Available from: http://www.ema.europa.eu/ema/ index.jsp?curl=pages/medicines/human/referrals/Protelos_and_Osseor/ human_referral_prac_000025.jsp\&mid $=$ WC0b01ac05805c516f. Accessed July 3, 2014.

11. Abrahamsen B, Grove EL, Vestergaard P. Nationwide registry-based analysis of cardiovascular risk factors and adverse outcomes in patients treated with strontium ranelate. Osteoporos Int. 2014;25(2):757-762.
International Journal of Women's Health

\section{Publish your work in this journal}

The International Journal of Women's Health is an international, peerreviewed open-access journal publishing original research, reports, editorials, reviews and commentaries on all aspects of women's healthcare including gynecology, obstetrics, and breast cancer. The manuscript management system is completely online and includes

\section{Dovepress}

a very quick and fair peer-review system, which is all easy to use. Visit http://www.dovepress.com/testimonials.php to read real quotes from published authors. 